
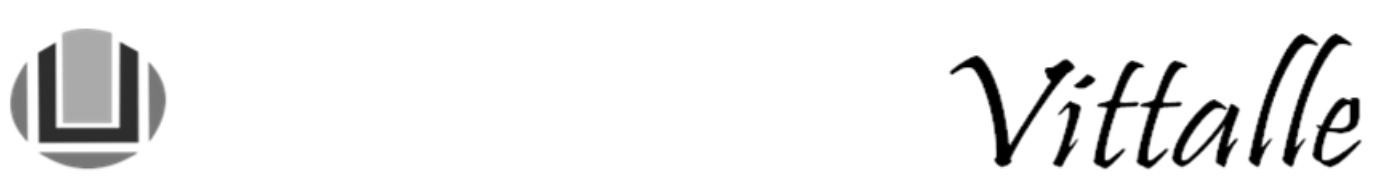

\title{
Canabinoides e Epilepsia: potencial terapêutico do canabidiol
}

\author{
Cristiane Ribeiro de Carvalho ${ }^{\mathrm{a}^{*}}$, Alexandre Ademar Hoeller ${ }^{\mathrm{b}}$, Pedro Leite Costa Franco ${ }^{\mathrm{c}}$, Ingrid \\ Eidt $^{\mathrm{c}}$, Roger Walz ${ }^{\mathrm{a}, \mathrm{b}, \mathrm{c}}$
}

aPrograma de Pós Graduação em Neurociências, Centro de Ciências Biológicas, Laboratório de Ciências Médicas, Universidade Federal de Santa Catarina, Florianópolis, SC, 88040-970, Brasil.

bPrograma de Pós Graduação em Ciências Médicas, Centro de Ciências da Saúde, Hospital Universitário, Laboratório de Ciências Médicas, Universidade Federal de Santa Catarina, Florianópolis, SC, 88040-970, Brasil.

'Departamento de Clínica Médica, Centro de Ciências da Saúde, Hospital Universitário, Laboratório de Ciências Médicas, Universidade Federal de Santa Catarina, Florianópolis, SC, 88040-970, Brasil.

\section{Histórico do Artigo}

Recebido em: 01/11/2016

Aceito em: 04/01/2017

\section{Palavras-chave \\ Canabinoides \\ Canabidiol \\ Cannabis medicinal \\ Epilepsia refratária \\ Anticonvulsivante.}

Keywords

Cannabinoid

Cannabidiol

Medical Cannabis

Refractory epilepsy

Anticonvulsant

*Autor correspondente: decarvalhocr@gmail.com (C.R. de Carvalho)

\section{RESUMO}

Diversas evidências a respeito do potencial terapêutico dos dois compostos majoritários presentes nas plantas do gênero Cannabis - canabidiol (CBD) e $\Delta$-9-tetraidrocanabinol - indicam sua relevância clínica no tratamento das epilepsias. Extratos padronizados com alto teor de canabidiol tem se mostrado eficazes na redução da frequência e severidade das convulsões, particularmente em crianças com tipos raros de epilepsia que são refratárias aos fármacos convencionais. Tais evidências têm motivado a regulamentação do uso clínico de extratos padronizados contendo canabidiol para tratamento de casos graves de epilepsia no Brasil. Nesse sentido, a presente revisão traz um apanhado geral sobre a farmacologia do sistema endocanabinoide, os medicamentos baseados em Cannabis disponíveis para uso clínico, bem como o uso do canabidiol no tratamento das epilepsias em humanos. Importante ressaltar, que apesar dos resultados promissores, ainda existem poucos estudos clínicos bem delineados que garantam a eficácia, segurança e tolerabilidade do CBD no tratamento de pacientes com epilepsia intratável, tais como nas síndromes de Dravet e Lennox-Gastaut.

\section{Cannabinoids and epilepsy: potential therapeutic role of cannabidiol}

ABSTRACT - There are many evidences about the therapeutic potential of two major compounds present in plants of the genus Cannabis - cannabidiol (CBD) and $\Delta$-9-tetrahydrocannabinol especially in relation to their clinical relevance in the treatment of epilepsy. Anecdotally, standardized extracts with a high content of cannabidiol has proven to be effective in reducing the frequency and severity of seizures, particularly in children with rare types of epilepsy which are refractory to conventional antiepileptic drugs. These findings have encouraged the regulation of clinical use of standardized extracts containing cannabidiol for treatment of severe epilepsy in Brazil. In this sense, this review provides an overview of the pharmacology of the endocannabinoid system, drug basedCannabis available for clinical use, as well as, cannabidiol for epilepsy treatment. Important to note, despite some promising results, small and methodologically limited studies of CBD in human epilepsy have been inconclusive. Consequently, there are still few well-designed clinical studies to ensure the effectiveness, safety and tolerability of the CBD in the treatment of patients with intractable epilepsy, such as observed in the Dravet and Lennox-Gastaut syndromes. 


\section{Introdução}

A epilepsia é uma das principais doenças neurológicas que acometem adultos jovens no mundo. Caracteriza-se pela ocorrência espontânea e recorrente de episódios breves ou prolongados de atividade neuronal excessiva, devido a um estado de hiperexcitabilidade neuronal e hipersincronia (1). Essas alterações nas descargas neuronais geram crises, que podem ser localizadas, compreendendo um dos hemisférios cerebrais (crises parciais ou focais) ou difusas, quando ambos os hemisférios são atingidos (crises generalizadas). As crises podem se manifestar de diferentes maneiras, dependendo do estado de consciência do indivíduo e do comprometimento do hemisfério afetado (2). O tratamento farmacológico das epilepsias objetiva interromper as crises através da administração de fármacos anticonvulsivantes, embora o tratamento seja ineficaz em até $30 \%$ dos pacientes (3). Atualmente, diversos fármacos (cerca de 25) estão disponíveis para o tratamento de pacientes com epilepsia. Apesar desse arsenal terapêutico, não houve grande avanço em relação à eficácia terapêutica considerando os pacientes refratários à medicação, embora alguns benefícios tenham sido obtidos em outros desfechos clínicos (por exemplo, melhor tolerabilidade e menor interação medicamentosa) (4). Em virtude disso, existe uma demanda no campo científico para o desenvolvimento de novos fármacos anticonvulsivantes que sejam mais eficazes no tratamento de casos refratários e tenham um perfil de segurança favorável (4). Nesse cenário, os derivados canabinoides estão ganhando espaço, uma vez que apresentam um mecanismo de ação distinto dos fármacos anticonvulsivantes convencionais e parecem ter efeitos colaterais bem tolerados pelos pacientes $(5,6)$.

Entre os canabinoides presentes na Cannabis, o mais estudado é o canabidiol (CBD), devido à sua propriedade anticonvulsivante e, especialmente, pela ausência de efeitos psicotomiméticos e risco de desenvolvimento de dependência típicos do $\Delta-9$ tetraidrocanabinol (THC) (5). Curiosamente, o THC age como agonista parcial dos receptores canabinoides $\mathrm{CB} 1$ e $\mathrm{CB} 2$ e exerce efeitos pró-convulsivantes ou anticonvulsivantes, dependendo da dose e do modelo experimental utilizado (7). Recentemente, foi demostrado que a administração central de um agonista seletivo dos receptores CB2 (AM1241) pode aumentar a severidade de convulsões induzidas farmacologicamente em ratos (8).

Evidências experimentais indicam que os efeitos anticonvulsivantes do THC ou análogos sintéticos são mediados pela ativação dos receptores de canabinoides $\mathrm{CB} 1$, ao passo que os efeitos do CBD parecem mediados por um mecanismo independente da sinalização endocanabinoide (9). Ademais, os estudos pré-clínicos revelaram que o THC pode promover efeitos ambíguos, podendo inibir convulsões ou estimular atividades epileptiformes ou induzir convulsões, dependendo da dose e do modelo experimental utilizado. Em contrapartida, o CBD não apresenta esse efeito dual (10). A diferença de resultados apresentada entre esses estudos pode ser justificada, em parte, pela grande variabilidade de métodos usados, o emprego de diferentes modelos de indução de epilepsia ou convulsão, bem como a utilização de diferentes espécies de roedores, que podem metabolizar de forma distinta os fármacos testados, alterando seus efeitos (11). Além disso, é importante ressaltar que a maioria dos estudos realizados foram conduzidos com modelos animais num período agudo, havendo poucas investigações em modelos de epilepsia (crônicos), que tipicamente mostram-se mais translacionais e mimetizem melhor a doença $(5,6)$. Apesar das diversas evidências experimentais acerca das propriedades anticonvulsivantes dos canabinoides, particularmente do THC e do CBD, ainda existem poucos estudos que comprovem a sua eficácia e segurança em humanos $(5,6,12)$.

Anedoticamente, os extratos padronizados com alto teor de CBD tem se mostrado eficazes na redução da frequência e severidade das convulsões, particularmente em crianças com 
tipos raros de epilepsia que são refratárias à farmacoterapia convencional $(5,6,12)$. Essas evidências têm motivado a regulamentação do uso clínico, particularmente de extratos padronizados contendo CBD e THC, para o tratamento de casos graves de epilepsia no Brasil (13). Dado o exposto, a presente revisão traz um apanhado geral dos medicamentos baseados em Cannabis disponíveis para uso clínico e tem como enfoque o atual estado da arte do potencial terapêutico do canabidiol para tratamento da epilepsia.

\section{Material e métodos}

A presente revisão de literatura baseou-se em bancos de dados como PubMed e Google Scholar, além de consultas às diretrizes atuais do Ministério de Saúde, durante o período de Setembro e Outubro de 2016. Foram usadas como palavras-chave: "canabinoide", "canabidiol" e "epilepsia". O foco da pesquisa foram artigos que abordam o uso medicinal de canabinoides, em especial no tratamento das epilepsias, seus mecanismos, efeitos colaterais e variedades de produtos farmacêuticos baseados em Cannabis disponíveis para uso clínico. Os artigos selecionados incluíram estudos transversais, revisões de literatura e notas técnicas disponíveis às comunidades terapêuticas sobre os fármacos em discussão no texto.

\section{Resultados e discussão}

\section{Os Canabinoides e o Sistema Endocanabinoide}

O gênero Cannabis, parte da família Cannabaceae, é conhecido desde 4000 A.C., data das primeiras evidências do cultivo de fibras da planta na China, durante a dinastia Han. Há indícios do uso medicinal, recreacional e religioso da planta a partir de 1000 A.C na Índia, Tibete, Pérsia e Assíria, logo atingindo a Europa pelo Mediterrâneo e persistindo até os tempos modernos. Após um período de decréscimo no uso Cannabis no início do século $\mathrm{XX}$, seguido de um processo mundial de restrição do seu uso e cultivo, iniciado nos EUA, um novo interesse pela planta surgiu com a descoberta dos primeiros fitocanabinoides (14).

Os canabinoides constituem um grupo heterogêneo de substâncias endógenas e exógenas que exercem diversas ações farmacológicas através da interação com o sistema endocanabinoide. Existem três classes de canabinoides: fitocanabinoides, endocanabinoides e canabinoides sintéticos. As plantas do gênero Cannabis contém mais de 100 compostos relacionados quimicamente e biossinteticamente, as quais são coletivamente denominadas fitocanabinoides (15). Os fitocanabinoides são compostos terpenoides lipofílicos derivados do resorcinol, que são estruturalmente distintos, porém farmacologicamente semelhantes, aos ligantes endógenos (endocanabinoides) canabinoides sintéticos. O THC e o CBD são os dois compostos majoritários da Cannabis e os principais responsáveis pelas ações farmacológicas da planta. Entretanto, outros fitocanabinoides como: canabigerol, canabicromeno, canabivarina, tetrahidrocanabivarina, canabicromevarina, canabigerovarina também podem exercer potenciais efeitos terapêuticos, conforme já demonstrado em estudos pré-clínicos e clínicos (15,16). A descrição dos fitocanabinoides mais conhecidos, notadamente o THC, data do começo dos anos 70, seguido pelo CBD e iniciando nesse período um fervor por estudar tais substâncias. Depois do período lento de publicações no final dos anos 70, um novo interesse surgiu após a descoberta do sistema endocanabinoide nos anos 90, além da descrição de seus receptores e o isolamento da anandamida, um dos principais ligantes endógenos e integrados à fisiologia humana em diversos processos (17).

$\mathrm{O}$ sistema endocanabinoide exerce funções reguladoras importantes, particularmente no sistema nervoso central (SNC) e imunológico, tornando-o um alvo terapêutico potencial para vários distúrbios, incluindo náuseas e vômito, dor, inflamação, doenças 
cardiovasculares, glaucoma, câncer, espasticidade e epilepsia. O sistema endocanabinoide consiste basicamente de receptores CB1 e CB2, ligantes endógenos, além de enzimas de síntese e degradação (18). Os receptores canabinoides pertencem à superfamília dos receptores de membrana acoplados à proteína $\mathrm{G}$ e a ativação desses receptores promove a supressão da excitabilidade neuronal e inibição da liberação de vários neurotransmissores, incluindo monoaminas, aminoácidos excitatórios e inibitórios, além de neuropeptídeos (19). Os principais ligantes endógenos dos receptores canabinoides são a etanolamina do ácido araquidônico (anandamida) e o 2-araquidonilglicerol (2-AG), os quais derivam de ácidos graxos poliinsaturados de cadeia longa, particularmente do ácido araquidônico. Contudo, outras moléculas lipídicas, tais como oleamida, O-araquidonoil etanolamina (virodamina) 2araquiidonoil gliceril éter (noladina) e $\mathrm{N}$-araquidonoil-dopamina (NADA) também possuem ação canabimimética. Os endocanabinoides são produzidos sob demanda nos neurônios póssinápticos e agem como mensageiros retrógrados em terminais pré-sinápticos neuronais (20) (Figura 1). Assim como, podem agir de maneira não-retrógrada e também autócrina (sinalização neurônio-astrócito) $(15,21)$. Os receptores canabinoides do tipo CB1 e CB2 são receptores acoplados à proteína $\mathrm{G}$ inibitória, que inibem atividade da adenilato ciclase reduzindo os níveis de AMP cíclico. Ambos os receptores canabinoides CB1 e CB2 exercem seus efeitos através de múltiplos efetores intracelulares e possuem mecanismos de transdução de sinais distintos (22).

Os receptores CB1 são amplamente expressos no SNC e apresentam maior densidade de expressão em áreas que estão associadas com os seus efeitos característicos do THC, particularmente no prejuízo da cognição, aprendizagem e memória (amígdala, córtex e hipocampo), função motora e do movimento (gânglios da base e cerebelo), motivação/reforço e risco de desenvolver a dependência (via mesocorticolímbica, particularmente a área tegmentar ventral e núcleo accumbens) (23).

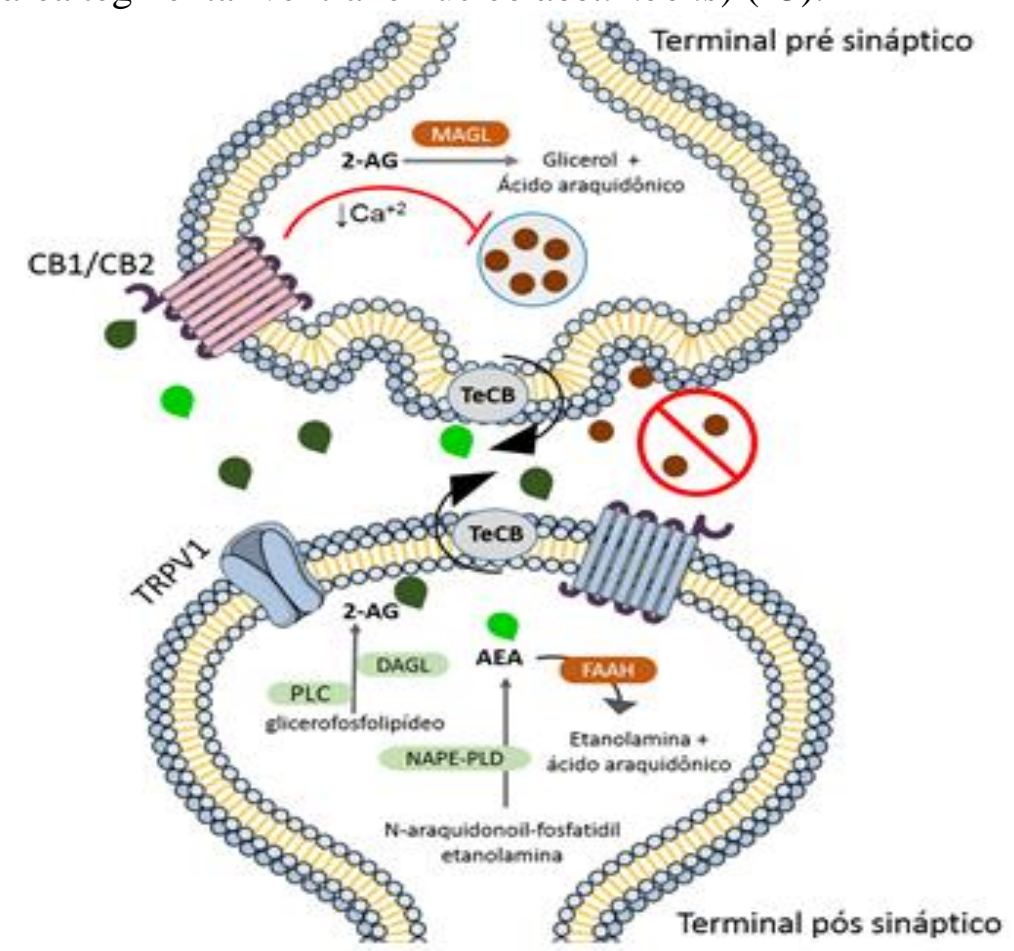

Figura 1: Representação simplificada do sistema endocanabinoide. Os endocanabinoides (eCBs), como o 2AG e anandamina (AEA), são sintetizados nos neurônios pós-sinápticos e se difundem, pela fenda sináptica, onde agem sobre os receptores canabinoides nos neurônios pré-sinápticos. Conforme demonstrado, o 2-AG é sintetizado em neurônios pós-sináptico através das enzimas fosfolipase C (PLC) e diacilglicerol lipase (DAGL), as quais se localizam principalmente na membrana plasmática, sendo então catabolizado pela enzima monoacilglicerol lipase (MAGL), localizada em neurônios pré-sinápticos. A biossíntese da anandamina 
também ocorre em neurônios pós-sinápticos, principalmente pela ação da enzima N-acil-fosfatidiletanolamina fosfolipase D (NAPE-LPD), e o seu catabolismo ocorre através da amidohidrolase de ácidos graxos (FAAH, sigla em inglês), que se localiza principalmente em neurônios pré-sinápticos. Os transportadores de membrana dos eCBs (TeCB) localizados em neurônios pré e pós-sinápticos facilitam a recaptação dos eCBs. A ativação dos receptores canabinoides causa uma redução da excitabilidade do neurônio pré-sináptico, através da despolarização, com consequente redução da liberação de neurotransmissores (A Figura 1 foi elaborada pelo autor através da plataforma Mind the Graph / www.mindthegraph.com).

Os principais efeitos fisiológicos dos endocanabinoides, bem como dos ligantes exógenos (incluindo o THC) são mediados pela ativação dos receptores CB1 (24), expressos predominantemente em terminais pré-sinápticos de neurônios GABAérgicos e glutamatérgicos (25), onde modulam formas mais persistentes de plasticidade sináptica, seja pela indução da depressão de longa duração (LTD, do inglês long-term depression) (19) ou indiretamente, ao induzir a LTP (do inglês, long-term potentiation) (26). Coletivamente, esses mecanismos de plasticidade contribuem com processos de aprendizagem, memória e dependência e no controle da hiperexcitabilidade neuronal $(27,28)$.

Os receptores CB2 estão localizados predominantemente em células imunes, sendo tradicionalmente referidos como receptores canabinoides periféricos. Contudo, recentemente os receptores CB2 foram identificados em células microgliais, astrócitos e em determinadas subpopulações de neurônios no SNC em condições fisiológicas normais (29). Ademais, foi observado um aumento na expressão dos receptores CB2 em humanos diagnosticados com doença de Alzheimer, esclerose múltipla, esclerose amiotrófica lateral e doença de Parkinson (30, 31). Tal observação indica que a supra-regulação dos receptores CB2 caracteriza um padrão de resposta frente a diferentes tipos de "lesão crônica" ao SNC, possivelmente relacionada ao componente neuroinflamatório das doenças neurodegenerativas (30).

\section{Canabinoides : Uso Recreacional e Dependência}

A maconha é a terceira droga de abuso mais consumida no mundo, atrás do álcool e do tabaco. Estima-se que aproximadamente 3,8\% da população global consome Cannabis pelo menos uma vez por ano (32). A dependência de Cannabis é considerada um transtorno psiquiátrico moderado, sendo que, um em cada nove usuários de maconha atende os critérios de dependência (33). Além disso, o número de indivíduos que procuram tratamento para deixar de fumar maconha tem aumentado, sobretudo, na Europa e nos EUA (34).

Os efeitos reforçadores (sensação de bem estar, euforia e relaxamento), bem como os efeitos psicotomiméticos e as alterações cognitivas relacionadas ao uso de Cannabis são mediadas pela ativação dos receptores canabinoides CB1. Uma das principais limitações para o desenvolvimento terapêutico de fármacos que ativam diretamente os receptores CB1 é risco de desenvolver dependência e sintomas psicóticos (24). Curiosamente, um estudo sugeriu que o consumo de Cannabis em pacientes epilépticos não afeta o curso da doença, mas pode exacerbar as crises em indivíduos epilépticos com histórico de uso de outras drogas de abuso (35).

\section{Canabinoides de Uso Medicinal}

O termo "Cannabis medicinal" ou "maconha medicinal" refere-se ao uso de partes da Cannabis ou de canabinoides derivados da planta para tratar ou aliviar os sintomas (dor, espasticidade, náuseas e vômitos) de uma doença específica. Alguns países, tais como Canadá, EUA e Holanda, dispõem de produtos herbais, preparações farmacêuticas derivadas de Cannabis para uso medicinal, bem como medicamentos alopáticos (Tabela 1). 
Tabela 1. Canabinoides derivados da Cannabis e produtos sintéticos disponíveis para uso medicinal.

\begin{tabular}{|c|c|c|c|c|}
\hline Canabinoides & $\begin{array}{c}\text { Nome } \\
\text { comercial }\end{array}$ & $\begin{array}{c}\text { Vias de } \\
\text { administração }\end{array}$ & $\begin{array}{r}\text { Indicação } \\
\text { terapêutica }\end{array}$ & $\begin{array}{c}\text { País em que se encontra } \\
\text { disponível }\end{array}$ \\
\hline $\begin{array}{r}22 \%:<1 \% \\
\text { (THC: } \mathrm{CBD})\end{array}$ & Bedrocan ${ }^{\circledR}$ & $\begin{array}{l}\text { Vaporização, } \\
\text { óleo, chá }\end{array}$ & $\begin{array}{l}\text { Náuseas, vômitos, } \\
\text { anorexia, glaucoma }\end{array}$ & $\begin{array}{l}\text { Canadá, Holanda, } \\
\text { Alemanha, Itália, Finlândia }\end{array}$ \\
\hline $\begin{array}{l}13,5 \%:<1 \% \\
\text { (THC: CBD) }\end{array}$ & Bedrobinol $®$ & $\begin{array}{l}\text { Vaporização, } \\
\text { óleo, chá }\end{array}$ & $\begin{array}{l}\text { Náuseas, vômitos, } \\
\text { anorexia, glaucoma }\end{array}$ & $\begin{array}{l}\text { Canadá, Holanda, } \\
\text { Alemanha, Itália, Finlândia }\end{array}$ \\
\hline $\begin{array}{c}14 \%:<1 \% \\
(\mathrm{THC}: \mathrm{CBD})\end{array}$ & Bedica $®$ & $\begin{array}{l}\text { Vaporização, } \\
\text { óleo, chá }\end{array}$ & $\begin{array}{l}\text { Náuseas, vômitos, } \\
\text { anorexia, glaucoma }\end{array}$ & $\begin{array}{c}\text { Canadá, Holanda, } \\
\text { Alemanha, Itália, Finlândia }\end{array}$ \\
\hline $\begin{array}{c}6,5 \%: 8 \% \\
\text { (THC: CBD) }\end{array}$ & Bediol® & $\begin{array}{l}\text { Vaporização, } \\
\text { óleo, chá }\end{array}$ & $\begin{array}{l}\text { Dor neuropática, } \\
\text { doenças } \\
\text { inflamatórias, } \\
\text { epilepsia }\end{array}$ & $\begin{array}{l}\text { Canadá, Holanda, } \\
\text { Alemanha, Itália, Finlândia }\end{array}$ \\
\hline $\begin{array}{c}0,4 \%: 9 \% \\
\text { (THC: CBD) }\end{array}$ & Bedrolite ${ }^{\circledR}$ & $\begin{array}{l}\text { Vaporização, } \\
\text { óleo, chá }\end{array}$ & $\begin{array}{l}\text { Dor neuropática, } \\
\text { doenças } \\
\text { inflamatórias, } \\
\text { epilepsia }\end{array}$ & $\begin{array}{l}\text { Canadá, Holanda, } \\
\text { Alemanha, Itália, Finlândia }\end{array}$ \\
\hline $\begin{array}{l}\text { Diferentes } \% \text { e } \\
\text { proporções de } \\
\text { THC e CBD }\end{array}$ & Cannimed $\AA$ & $\begin{array}{l}\text { Vaporização, } \\
\text { óleo }\end{array}$ & Dores inflamatórias & Canadá \\
\hline $\begin{array}{c}2,7 \mathrm{mg} \text { de } \mathrm{THC} \\
\text { e } 2,5 \mathrm{mg} \text { (por } \\
\mu \mathrm{l})\end{array}$ & Sativex $₫$ & $\begin{array}{l}\text { Oromucosa } \\
\text { (spray) }\end{array}$ & $\begin{array}{l}\text { Dores neuropáticas } \\
\text { e inflamatórias }\end{array}$ & Reino Unido \\
\hline $\begin{array}{l}\text { Dronabinol } \\
(2,5-10 \\
\text { mg/cps })\end{array}$ & Marinol® & Oral (cápsulas) & $\begin{array}{l}\text { Náuseas, vômitos, } \\
\text { anorexia relacionada } \\
\text { à AIDS }\end{array}$ & $\begin{array}{c}\text { EUA, Austrália, Nova } \\
\text { Zelândia, Alemanha, África } \\
\text { do Sul }\end{array}$ \\
\hline $\begin{array}{l}\text { Nabilona } \\
\text { (1mg/cps) }\end{array}$ & Cesamet ${ }^{\circledR}$ & Oral (cápsulas) & Náuseas, vômitos & $\begin{array}{l}\text { EUA, Canadá, Alemanha, } \\
\text { Austrália, Reino Unido }\end{array}$ \\
\hline $\begin{array}{r}0 \%: 98 \% \\
\text { (THC: CBD) }\end{array}$ & Epilodex ${ }^{\circledR}$ & Solução oral & $\begin{array}{l}\text { Epilepsias raras (ex. } \\
\text { Síndromes de } \\
\text { Lennox-Gastaut e } \\
\text { Dravet) }\end{array}$ & $\begin{array}{c}\text { Estudos Clínicos } \\
\text { multicêntricos em fase III * }\end{array}$ \\
\hline
\end{tabular}

(THC : CBD) indicam as respectivas proporções de $\Delta$-9-tetrahidrocanabinol e canabidiol, em porcentagem, que está presente nas diferentes formulações. (*) fonte https://clinicaltrials.gov.

Os produtos herbais - derivados de plantas do gênero Cannabis cultivadas em condições específicas e controladas - são produtos padronizados e purificados (livres de contaminantes químicos e microbiológicos), os quais diferem entre si principalmente em relação à concentração de THC e CBD presente na formulação $(36,37)$. O primeiro medicamento fitoterápico derivado da Cannabis disponível para uso clínico desde 2005 foi o nabiximols (Sativex ${ }^{\circledR}$ ). Este medicamento consiste em um extrato alcoólico da Cannabis que contêm proporções quase equimolares de THC e CBD, disponível na forma de spray oral, utilizado no tratamento de espasticidade em pacientes com esclerose múltipla e no alívio de dores neuropática e oncológica em alguns países (38).

Ademais, já existem medicamentos alopáticos contendo agonistas canabinoides sintéticos como o dronabinol e nabilona, disponíveis para uso clínico em alguns países. O dronabinol é 
um isômero do THC, enquanto a nabilona possui estrutura química análoga ao THC. Ambos os fármacos exercem seus efeitos terapêuticos através da ativação dos receptores canabinoides. Em geral, eles são utilizados como estimulantes do apetite em pacientes com anorexia associada à AIDS, no alívio de náuseas e vômitos induzidas por quimioterapia oncológica refratária aos fármacos convencionais ou ainda como analgésico adjuvante em dores neuropáticas (37). O uso medicinal de produtos herbais derivados da Cannabis é bastante controverso, em parte devido a falta de padronização entre os produtos que assegurem a segurança e a dosagem consistente e, em parte, devido a questões envolvendo o aspectos legais (37).

Outros fatores que limitam o uso de produtos herbais derivados da Cannabis na prática clínica são informações divergentes sobre a proporção ideal de THC e CBD nas preparações, o seu perfil de segurança e quais doses (THC e CBD) deveriam ser usadas para se atingir o efeito terapêutico desejado (6). Vale a pena ressaltar que não existem estudos consistentes que comprovem a segurança da Cannabis medicinal fumada, apesar do uso de vaporizadores minimizar os riscos da exposição às toxinas presentes na fumaça inalada que contém algumas substâncias tóxicas também encontradas no tabaco. Esse risco deve ser considerado principalmente em relação aos pacientes que fazem uso prolongado de inaladores sob o risco desenvolverem faringite, rinite, asma, bronquite, enfisema e câncer de pulmão (39).

Em geral, os (agonistas) canabinoides de uso clínico produzem efeitos adversos, similares àqueles observados pelos usuários recreacionais de Cannabis (Quadro 1) (40). Alterações psiquiátricas também foram relatadas após o uso de canabinoides, incluindo psicose e ansiedade, sendo estes sintomas relacionados a concentrações mais elevadas de THC (24, 38). É importante enfatizar que o perfil de efeitos colaterais do CBD não são os mesmos esperados com uso do THC e seus análogos (38). Além disso, o risco de desenvolver dependência poderia ser uma fator limitante para o uso clínico, particularmente, dos agonistas dos receptores CB1 (6).

Quadro 1. Principais efeitos adversos dos canabinoides de uso clínico

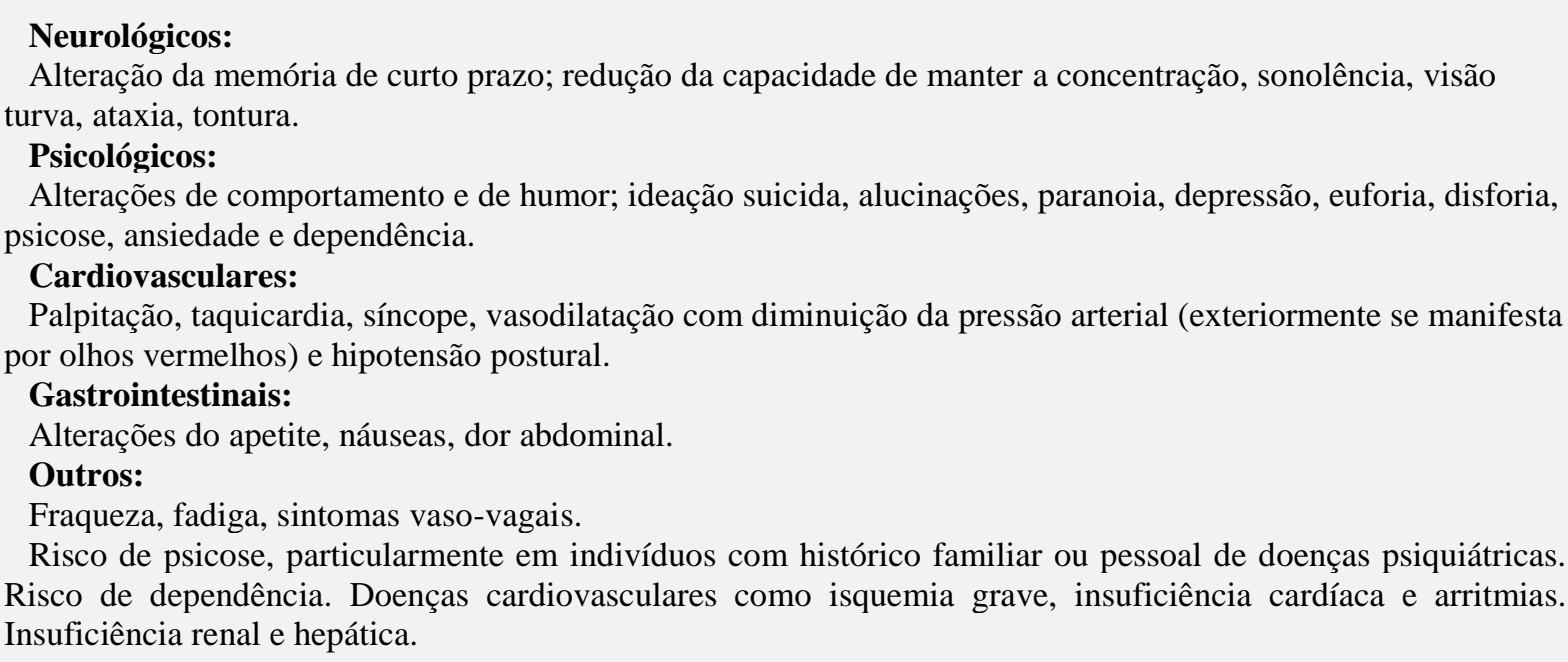

\section{Efeitos do Canabidiol em Pacientes com Epilepsia}

O primeiro estudo clínico que demonstrou o efeito anticonvulsivante do canabidiol foi conduzido no Brasil, pelo grupo do renomado pesquisador Dr. Elisaldo Carlini. Esse estudo duplo-cego foi realizado com 15 pacientes que sofriam pelo menos uma crise generalizada por semana, mesmo recebendo algum outro anticonvulsivante (fenitoína, primidona, clonazepam, carbamazepina, trimetadiona e /ou etossuximida) (41). No total, 8 pacientes receberam entre $200-300 \mathrm{mg} / \mathrm{dia}$ de CBD puro por via oral, durante 8 semanas. Destes 
pacientes, apenas um não obteve nenhuma melhora clínica. Entre os demais, quatro tiveram as convulsões totalmente abolidas durante o período em que tomaram CBD e três tiveram redução significativa na frequência das crises. No grupo de pacientes que recebeu placebo junto com seu outro anticonvulsivante, apenas um demonstrou melhora. Entretanto, não há avaliação do efeito do CBD na ausência de qualquer outro anticonvulsivante, mas o estudo sugeriu que o CBD poderia ser um adjuvante no tratamento da epilepsia (42).

\section{Considerações finais}

Ainda que o uso de canabidiol para tratamento de epilepsias refratárias à farmacoterapia convencional não seja mais uma novidade, mais estudos ainda são necessários para indicar livremente o seu uso. Por razões diversas, entre elas o número limitado de pacientes em cada um dos estudos clínicos, não se pode concluir com certeza a sua eficácia isolada ou se somente teria a capacidade de potencializar o efeito das outras drogas. Em revisão da renomada base de dados Cochrane, observou-se que não existem evidências confiáveis no momento para advogar pelo uso do CBD no tratamento da epilepsia, sugerindo a realização de estudos clínicos mais criteriosos (multicêntricos, randomizados, duplo-cegos e com maior duração) (10,47). Considerando a escassez de estudos clínicos em grande escala proporcionalmente ao longo período no qual os canabinoides são utilizados para fins medicinais, é necessário ponderar sobre o que está por trás da dificuldade de se realizar estudos mais criteriosos. É possível que aspectos da legislação (peculiar de cada país) assim como os efeitos indesejáveis associados aos canabinoides e finalmente o estigma gerado na sociedade em consequência ao uso recreacional da Cannabis possam ter contribuído em muito para isso.

Atualmente, não há registro de medicamento que contenha canabidiol ou outro canabinoide para uso clínico no Brasil. No entanto, em maio de 2015 a ANVISA publicou a resolução $\mathrm{N}^{\circ}$ 17, estabelecendo critérios e autorizando a importação de produtos à base de canabidiol para uso exclusivamente terapêutico em casos selecionados - desde que prescritos pelo médico legalmente habilitado (48). A academia brasileira de neurologia afirmou que os dados atualmente disponíveis na literatura não são suficientes para sustentar o uso de canabidiol como tratamento rotineiro na epilepsia, mas que este poderia ser efetivo em casos refratários ou de difícil tratamento. Reforçou ainda que precisam ser realizados mais estudos para estabelecer a segurança e a eficácia do canabidiol (49).

Conflito de interesses: Os autores declaram não haver conflito de interesses

\section{Referências}

1. French JA, Pedley TA. Clinical practice. Initial management of epilepsy. N Engl J Med. 2008 Jul 10;359(2):166-76.

2. Fisher R, Boas W, Blume W, Elger C, Genton P, Lee P et al. Epileptic Seizures and Epilepsy: Definitions Proposed by the International League Against Epilepsy (ILAE) and the International Bureau for Epilepsy (IBE). Epilepsia. 2005;46(4):470-472.

3. Dalic L, Cook M. Managing drug-resistant epilepsy: challenges and solutions. Neuropsychiatric Disease and Treatment. 2016; Volume 12:2605-2616.

4. Perucca P, Gilliam F. Adverse effects of antiepileptic drugs. The Lancet Neurology. 2012;11(9):792802.

5. Devinsky O, Cilio M, Cross H, Fernandez-Ruiz J, French J, Hill C et al. Cannabidiol: Pharmacology and potential therapeutic role in epilepsy and other neuropsychiatric disorders. Epilepsia. 2014;55(6):791-802.

6. Leo A, Russo E, Elia M. Cannabidiol and epilepsy: Rationale and therapeutic potential. Pharmacological Research. 2016;107:85-92. 
7. Friedman D, Devinsky O. Cannabinoids in the Treatment of Epilepsy. New England Journal of Medicine. 2015;374(1):94-95.

8. de Carvalho C, Hoeller A, Franco P, Martini A, Soares F, Lin K et al. The cannabinoid CB2 receptorspecific agonist AM1241 increases pentylenetetrazole-induced seizure severity in Wistar rats. Epilepsy Research. 2016;127:160-167.

9. Ibeas Bih C, Chen T, Nunn A, Bazelot M, Dallas M, Whalley B. Molecular Targets of Cannabidiol in Neurological Disorders. Neurotherapeutics. 2015;12(4):699-730.

10. Gloss D, Vickrey B. Cannabinoids for epilepsy. Cochrane Database Syst Rev. 2014;3:CD009270.

11. Reddy D, Golub V. The Pharmacological Basis of Cannabis Therapy for Epilepsy. Journal of Pharmacology and Experimental Therapeutics. 2016;357(1):45-55.

12. dos Santos RG, Hallak JE, Leite JP, Zuardi AW, Crippa JA. Phytocannabinoids and epilepsy. J Clin Pharm Ther. 2015 Apr; 40(2):135-43.

13. Resolução da Diretoria Colegiada - RDC no 17 de 06/05/2015. Disponível em http://portal.anvisa.gov.br/legislacao. Acesso em 20 de setembro de 2016.

14. Zuardi A. History of cannabis as a medicine: a review. Revista Brasileira de Psiquiatria. 2006;28(2):153-157.

15. Pertwee R. The diverse CB1and CB2receptor pharmacology of three plant cannabinoids: $\Delta 9$ tetrahydrocannabinol, cannabidiol and $\Delta 9$-tetrahydrocannabivarin. British Journal of Pharmacology. 2008;153(2):199-215.

16. Ligresti A, De Petrocellis L, Di Marzo V. From Phytocannabinoids to Cannabinoid Receptors and Endocannabinoids: Pleiotropic physiological and pathological roles through complex pharmacology. Physiol Rev. 2016 Oct; 96(4):1593-659.

17. Martin BR, Mechoulam R, Razdan RK. Discovery and characterization of endogenous cannabinoids. Life Sci. 1999;65(6-7):573-95.

18. Munro S, Thomas K, Abu-Shaar M. Molecular characterization of a peripheral receptor for cannabinoids. Nature. 1993;365(6441):61-65.

19. Wilson R. Endocannabinoid Signaling in the Brain. Science. 2002;296(5568):678-682.

20. Pamplona FA, Takahashi RN. Psychopharmacology of the endocannabinoids: far beyond anandamide. J Psychopharmacol. 2012 Jan;26(1):7-22.

21. Grueter BA, Brasnjo G, Malenka RC. Postsynaptic TRPV1 triggers cell type-specific long-term depression in the nucleus accumbens. Nat Neurosci. 2010 Dec; 13(12):1519-25.

22. Felder CC, Joyce KE, Briley EM, Mansouri J, Mackie K, Blond O, Lai Y, Ma AL, Mitchell RL. Comparison of the pharmacology and signal transduction of the human cannabinoid CB1 and CB2 receptors. Mol Pharmacol. 1995 Sep;48:443-450.

23. Di Marzo V. The endocannabinoid system: its general strategy of action, tools for its pharmacological manipulation and potential therapeutic exploitation. Pharmacol Res. 2009 Aug;60(2):77-84.

24. Volkow ND, Compton WM, Weiss SR. Adverse health effects of marijuana use. N Engl J Med. 2014 Aug 28;371(9):879

25. Svizenska I, Dubovy P, Sulcova A. Cannabinoid receptors 1 and 2 (CB1 and CB2), their distribution, ligands and functional involvement in nervous system structures - A short review. Pharmacology Biochemistry and Behavior. 2008;90(4):501-511.

26. Carlson G, Wang Y, Alger BE. Endocannabinoids facilitate the induction of LTP in the hippocampus. Nat Neurosci. 2002 Aug;5(8):723-4.

27. Sidhpura N, Parsons LH. Endocannabinoid-mediated synaptic plasticity and addiction-related behavior. Neuropharmacology. 2011 Dec;61(7):1070-87

28. Lutz B. On-demand activation of the endocannabinoid system in the control of neuronal excitability and epileptiform seizures. Biochemical pharmacology. 2004 Nov;68:1691-98.

29. Onaivi E, Ishiguro H, Gong J, Patel S, Perchuk A, Meozzi P et al. Discovery of the Presence and Functional Expression of Cannabinoid CB2 Receptors in Brain. Annals of the New York Academy of Sciences. 2006;1074(1):514-536.

30. Benito C, Tolón RM, Pazos MR, Núñez E, Castillo AI, Romero J. Cannabinoid CB2 receptors in human brain inflammation. Br J Pharmacol. 2008 Jan;153:277-285.

31. García M, Cinquina V, Palomo-Garo C, Rábano A, Fernández-Ruiz J. Identification of CB2 receptors in human nigral neurons that degenerate in Parkinson's disease. Neuroscience Letters. 2015;587:1-4.

32. UNODC, 2016: World Drug Report 2016. Disponível em https://www.unodc.org/wdr2016/en/cannabis. Acesso em 20 de setembro de 2016.

33. Lynskey MT, Heath AC, Bucholz KK, Slutske WS, Madden PA, Nelson EC, Statham DJ, Martin NG. Escalation of drug use in early-onset cannabis users vs co-twin controls. JAMA. 2003 Jan;2229;289(4):427-33. 
34. Murray RM, Morrison PD, Henquet C, Di Forti M. Cannabis, the mind and society: the hash realities. Nat Rev Neurosci. 2007 Nov;8(11):885-95.

35. Hamerle M, Ghaeni L, Kowski A, Weissinger F, Holtkamp M. Cannabis and other illicit drug use in epilepsy patients. European Journal of Neurology. 2013;21(1):167-170.

36. Pamplona F. Quais são e pra que servem os medicamentos à base de Cannabis?. Revista da Biologia. 2014; 13(1):28-35.

37. Whiting P, Wolff R, Deshpande S, Di Nisio M, Duffy S, Hernandez A et al. Cannabinoids for Medical Use. JAMA. 2015;313(24):2456.

38. Wang T, Collet J, Shapiro S, Ware M. Adverse effects of medical cannabinoids: a systematic review. Canadian Medical Association Journal. 2008;178(13):1669-1678.

39. Kalant H. Smoked Marijuana as Medicine: Not Much Future. Clin Pharmacol Ther. 2008;83(4):517519.

40. Bostwick JM. Blurred boundaries: the therapeutics and politics of medical marijuana. Mayo Clin Proc. 2012 Feb;87(2):172-86.

41. Cunha J, Carlini E, Pereira A, Ramos O, Pimentel C, Gagliardi R et al. Chronic Administration of Cannabidiol to Healthy Volunteers and Epileptic Patients. Pharmacology. 1980;21(3):175-185.

42. Trembly B, Sherman M. Double-blind clinical study of cannabidiol as a secondary anticonvulsant. Marijuana '90 International Conference on Cannabis and Cannabinoids; 1990 July 8-11; Kolympari, Crete. International Association for Cannabinoid Medicines, 1990:section 2-page 5.

43. Maa E, Figi P. The case for medical marijuana in epilepsy. Epilepsia. 2014;55(6):783-786.

44. NCT02091206 (número de identificação do estudo clínico). Double Blind, Placebo Controlled Twopart Study to Investigate the Dose-ranging Safety and Pharmacokinetics, Followed by the Efficacy and Safety of Cannabidiol (GWP42003-P) in Children and Young Adults WithDravet Syndrome. Disponívelem: ClinicalTrials.gov; Identifier (NCT02091206). Acesso em 7 de outubro de 2016.

45. NCT02224560(número de identificação do estudo clínico). A Study to Investigate the Efficacy and Safety of Cannabidiol (GWP42003-P; CBD) as Adjunctive Treatment for Seizures Associated With Lennox-Gastaut Syndrome in Children and Adult (GWPCARE3) Disponívelem: ClinicalTrials.gov; Identifier (NCT02224560). Acessoem 7 de outubro de 2016.

46. Devinsky O, Marsh E, Friedman D, Thiele E, Laux L, Sullivan J et al. Cannabidiol in patients with treatment-resistant epilepsy: an open-label interventional trial. The Lancet Neurology. 2016; 15(3):270-278

47. Throckmorton DC, 2015. Cannabidiol: Barriers to Research and Potential Medical Benefits. Disponível em: http://www.fda.gov/newsevents/testimony/ucm453989.htm. Acesso em 10 de setembro de 2016.

48. De Oliveira HC. O Uso da Substância Canabidiol (CBD) para o Tratamento da Epilepsia em Crianças. Nota Técnica No 02/2015. Disponível em: www.saude.mt.gov.br/arquivo/5035. Acesso em 10 de setembro de 2016.

49. Brucki S, Frota $\mathrm{N}$ et al. Cannabinoids in neurology - Brazilian Academy of Neurology. Arquivos de Neuro-Psiquiatria. 2015;73(4), 371-374. 